\title{
Melkersson-Rosenthal Syndrome Still a Mystery: A Case Report and Suggested Guideline Investigations
}

\author{
Manal Alsabbagh ${ }^{\mathrm{a}, \mathrm{b}}$, Azad Kassim ${ }^{\mathrm{a}}$
}

\begin{abstract}
Melkersson-Rosenthal syndrome (MRS) is a rare neuromucocutaneous syndrome of unknown etiology. Rarely, it presents with the full triad of relapsing facial swelling, facial palsy, and a fissured, geographic tongue. We present a case of a 22-year-old man with the three typical symptoms of MRS, which were managed successfully with systemic steroids. We highlight the potential etiologies of MRS and propose guidelines by which to investigate and subsequently identify the underlying cause of this mysterious disease.
\end{abstract}

Keywords: Etiology; Guideline; Investigations; Melkersson-Rosenthal syndrome

\section{Introduction}

Melkersson-Rosenthal syndrome (MRS), a rare disorder, may present with a triad of recurrent symptoms: orofacial swelling, relapsing facial palsy, and a fissured tongue [1]. The first two features were first reported by Melkersson in 1928, and the third, the fissured tongue, was reported by Rosenthal 3 years later [2]. Although allergic reactions, hypersensitivity, autoimmunity, genetic predisposition, and microbial reactions have been hypothesized to contribute to the pathogenesis of MRS [3], the actual cause has not yet been identified. The interval from presentation to diagnosis may be as great as three decades [4]. We present the case of a 22-year-old man with MRS who was misdiagnosed 2 years prior to the current presentation as having chronic allergic angioedema, which had partially responded to systemic corticosteroids.

Manuscript submitted June 29, 2018, accepted July 20, 2018

anternal Medicine Department, Dermatology, King Hamad University Hospital, Building 2345, Road 2835, Busaiteen 228, PO Box 24343, Kingdom of Bahrain

${ }^{b}$ Corresponding Author: Manal Alsabbagh, Internal Medicine Department, Dermatology, King Hamad University Hospital, Building 2345, Road 2835, Busaiteen 228, PO Box 24343, Kingdom of Bahrain.

Email: manal.alsabbagh@khuh.org.bh

doi: https://doi.org/10.14740/jmc3121w

\section{Case Report}

A 22-year-old Pakistani (Asian) man presented with a 4-day history of facial swelling. He was admitted to the Otorhinolaryngology Department with a provisional diagnosis of angioedema and nasal vestibulitis. As the patient failed to respond after an immediate dose of a systemic steroid (hydrocortisone) and a few days of systemic antibiotics (co-amoxiclav), the Dermatology Department was consulted.

A detailed history revealed that the patient had experienced five similar episodes within the last 2 years that sometimes occurred after eating chicken, red meat, or mutton. Additionally, he had been diagnosed with "recurrent" facial palsy 4 years previously. Some of these episodes were self-limited, whereas others required medical intervention. As those episodes occurred during visits to Pakistan, we were unable to determine what management was offered. The patient denied sensory dysfunction, fever, infections, history of insect bites, a positive family history, and being on any current medications.

Physical examination revealed gross facial asymmetry with a swollen right cheek and upper lip (Fig. 1a, b). The right forehead was smooth, the right nostril was crusted, and the upper lip was severely swollen, with a few firm, non-tender nodules. The swelling was painless and non-pitting. Examination of the oral cavity revealed gingival hyperplasia and a fissured, geographic tongue (Fig. 1c, d). Neurological examination showed right facial palsy (Fig. 1e, f). His sensation was intact, however, and cervical lymph nodes were not palpable.

Apart from a mildly elevated C-reactive protein level and low $\mathrm{C} 4$, the laboratory investigations were normal (Table 1). Histopathological examination of a punch biopsy specimen removed from the upper lip showed aggregations of inflammatory cells with dilated lymphovascular channels on a background of non-specific dermal edema (Fig. 2).

MRS was diagnosed on a clinical basis. The patient was started on a 1-week course of oral prednisolone $20 \mathrm{mg}$ once daily and fluticasone cream application twice daily. A course of co-amoxiclav was also completed. One week following discharge, the patient had residual facial edema and facial palsy with persistence of the fissured, geographic tongue. Further investigations were planned, but the patient did not return for the follow-up appointment.

\section{Discussion and Conclusions}

MRS is rare. The triad of suggestive symptoms (facial palsy, 

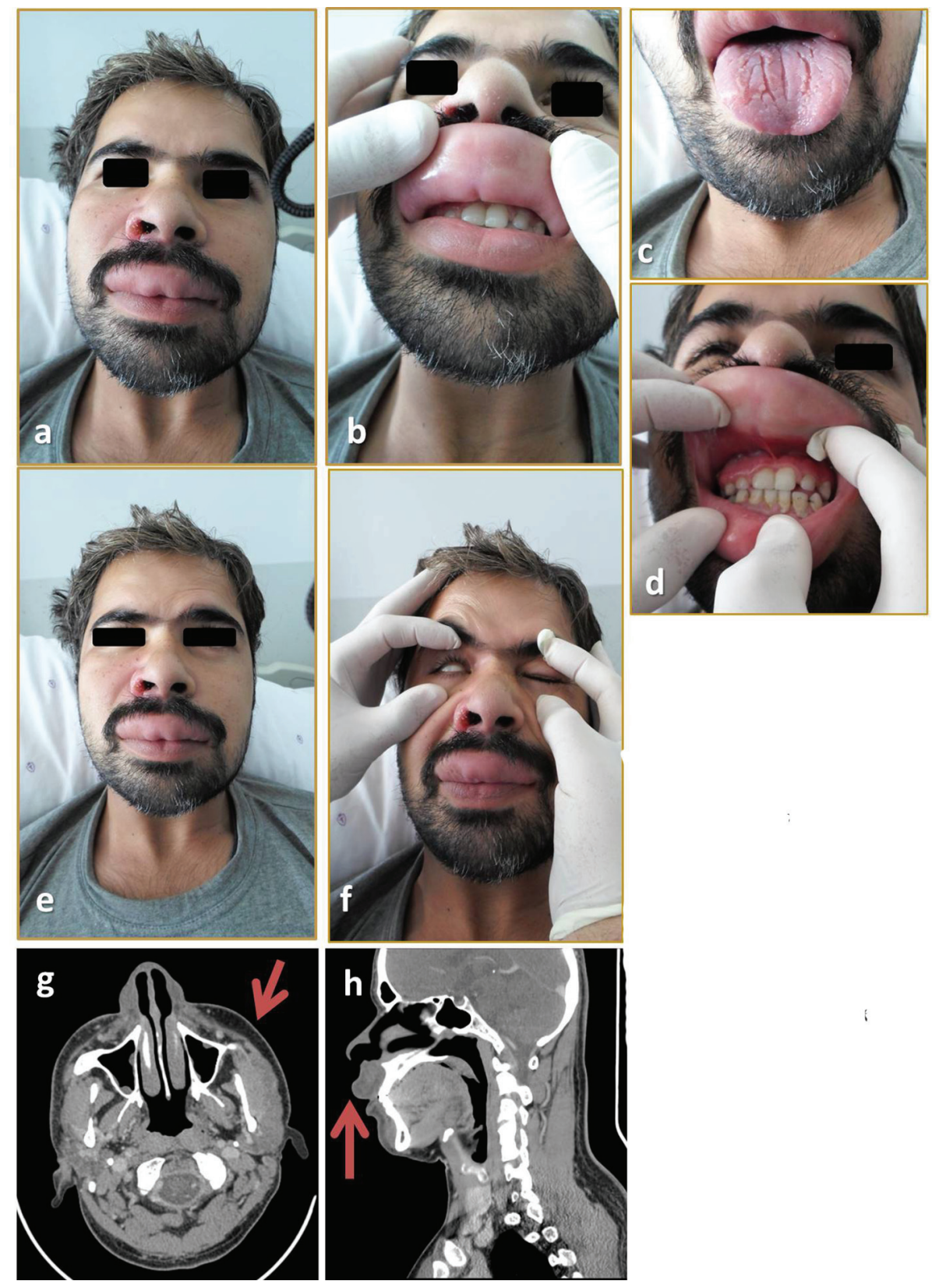

Figure 1. (a, b) Right side edema with swollen upper lip. (c, d) Fissured tongue, gingival hypertrophy. (e, f) Facial nerve examination; flattened right forehead, weakness in closing the right eye. $(g, h)$ CT scan showing right side facial swelling and upper lip swelling.

relapsing facial edema, and fissured tongue) were reported for only $8-18 \%$ of cases, and so the diagnosis is commonly overlooked [5]. Such cases typically present with facial palsy as the first complaint, as it generally precedes the other two symptoms by a few years [2, 6-8]. Hence, the triad of symptoms that would indicate a diagnosis of MRS is not consistently present [9]. We suggest that the sequence of the symptoms' onset may be indicative of the underlying pathology.

Physicians' interest and awareness of this syndrome seemed to have increased over the last two decades, as indicated in our literature search. For instance, our search for MRS in PubMed up to 2017 resulted in 848 publications, more than one-third of which were published within the last two decades. This large number of reported cases could facilitate a process for establishing an international guideline for the management of MRS, including diagnostic investigations and subsequent treatment.

\section{Etiology and investigations}

The proposed potential etiologies are based on the immunogenicity (allergy and autoimmunity - not yet proved) of the 
Table 1. Investigations

\begin{tabular}{ll}
\hline Investigation $^{\text {a }}$ & Results \\
\hline Complete blood count & Normal \\
CRP & Mildly elevated: $12.5 \mathrm{mg} / \mathrm{L}$ (normal: $0-3.0)$ \\
\hline ESR & Normalized after steroid course $(1.3 \mathrm{mg} / \mathrm{L})$ \\
Thyroid function tests & Normal \\
Anti-TG antibodies, anti-TPO antibodies & Normal \\
Renal function tests & Negative \\
TTG, anti-gliadin antibody & Normal \\
Anti-CCP & Negative \\
C3 & Negative \\
C4 & Normal \\
Antinuclear antibody & Mildly decreased. $0.13 \mathrm{~g} / \mathrm{L}$ (normal 0.17 - 0.52) \\
HIV & Negative \\
RAST for chicken meat, mutton, and beef & Negative \\
ACE level & Negative \\
Rheumatoid factor & Normal \\
\hline
\end{tabular}

ACE: angiotensin-converting antibody; C3, C4: third and fourth components of complement; HIV: human immunodeficiency virus; RAST: radioallergosorbent test; CRP: C-reactive protein; ESR: erythrocyte sedimentation rate; TG: thyroglobulin; TPO: thyroid peroxidase; TTG: tissue transglutaminase; CCP: cyclic citrullinated peptide; ANCA: anti-neutrophilic cytoplasmic antibody; PPD: purified protein derivative. aPlanned to be performed if symptoms of facial palsy persisted, but the patient missed the follow-up appointment: C1 esterase inhibitor, ANCA, immunoglobulin E, total iron profile, chest plain radiography, PPD (for tuberculosis), hepatitis C, serum cryoglobulins, patch test, head magnetic resonance imaging.

syndrome, associated infections, and the genetics of the patient. Morales et al found a lack of sensitization to food, additives, or contact in such cases [10]. In contrast, Fitzpatrick et al found that more than $50 \%$ of the patients had a positive allergy test, suggesting involvement of a type 1 hypersensitivity reaction in the development of orofacial granulomatosis [11]. The latter is consistent with the report by Feng et al, who found that $80 \%$ of 44 MRS patients exhibited an allergic response [12]. Although these findings suggest hypersensitivity as a potential etiologic factor, we are still unable to identify the type of hypersensitivity (type 1 vs. type 4 ) or to recommend a screening test.

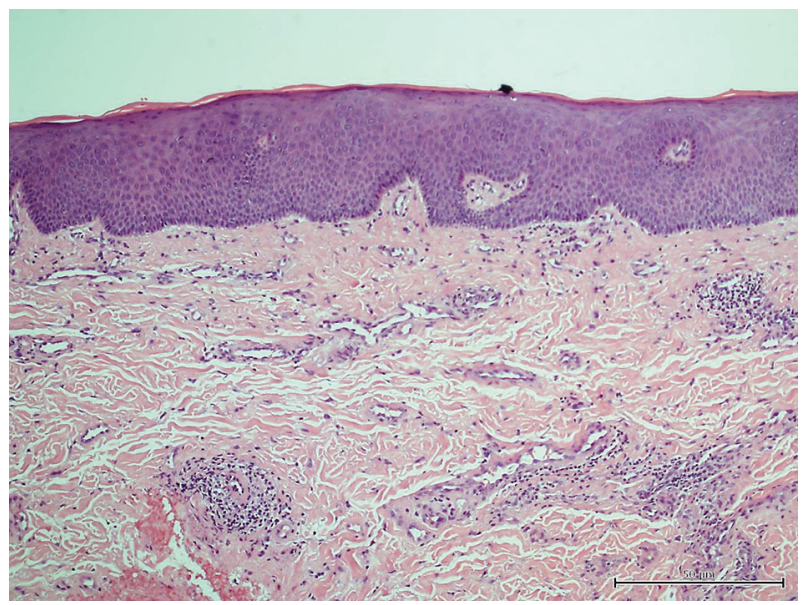

Figure 2. Biopsy from upper lip (mucosa).
Elevated immunoglobulin (Ig) levels were observed in some cases. Greene and Rogers reported increased IgM in three patients and increased IgE in one [2]. Liu and Yu found that $14 \%$ of MRS patients had elevated serum IgG, which suggests an associated immunologic derangement [13]. Although the association was documented in only a few cases, this finding should not be ignored.

The reported association between MRS and autoimmune thyroiditis as well as systemic lupus erythematosus favors the proposed theory of autoimmunity $[14,15]$. Crohn's disease is the only autoimmune disorder that has shown a strong association with MRS. The authors of a systematic review analysed the association between orofacial granulomatosis (OFG) and Crohn's disease in children concluded that $40 \%$ of patients with OFG would develop Crohn's disease during their lifetimes. As OFG has been proposed to be a subtype of Crohn's disease, the authors recommended long-term follow-up regardless of the current absence of systemic symptoms [16, 17]. Furthermore, Scully et al hypothesized that MRS, granulomatous cheilitis, and OFG are individual presentations of Crohn's disease or a condition very similar to it [18].

Complement deficiency was reported in a few MRS cases. Freeman reported an associated deficiency of $\mathrm{C} 1 \mathrm{q}$ along with a low-normal level of C4 and a positive pricked skin test [19]. A similar deficiency was reported in an MRS case associated with systemic lupus erythematosus [14]. Various complement types have been proposed to be responsible for these findings [19]. Masson et al measured C3, C4, and C1 esterase inhibitor levels and found that only two of seven MRS patients had 
Table 2. Suggested Guideline Investigations

CBC, ESR, CRP
Renal and liver function tests
Total immunoglobulins, subgroups
Thyroid function tests, thyroid antibodies
Anti-gliadin, TTG
C3, C4 levels
Antinuclear antibody, ANCA, renal function tests
C1 esterase inhibitor
ACE level
Chest plain radiography, PPD (for tuberculosis)
Biopsy to determine histopathology and to guide screening for microbial agents
Detailed history, including the first symptom of the triad to appear, number of years having the disease, when the biopsy was done, treatment
used, number of relapses

ACE: angiotensin-converting antibody; C3, C4: third and fourth components of complement; RAST: radioallergosorbent test; CRP: C-reactive protein; ESR: erythrocyte sedimentation rate; TTG: tissue transglutaminase; ANCA: anti-neutrophilic cytoplasmic antibody; PPD: purified protein derivative.

low $\mathrm{C} 1$ esterase inhibitor levels, although the $\mathrm{C} 3$ and $\mathrm{C} 4$ levels were normal [20]. To the best of our knowledge, our patient represents the third reported case of MRS with a low $\mathrm{C} 4$ level.

Troiano et al explored the role of infections and genetics in the MRS etiology [21]. The two main pathogens investigated were Mycobacterium tuberculosis and Borrelia burgdorferi. The skin biopsies were positive for $M$. tuberculosis but controversial for B. burgdorferi [21]. Trials of treating MRS with anti-tuberculosis regimens have not appeared in literature.

Exon sequencing was performed in a Chinese family in which some members had MRS that had been confirmed both clinically and histopathologically. Xu et al found that the fatty acid transport protein 1 (FATP1) gene had mutated in two affected members and a single unaffected member [22]. Prior to these findings, familial MRS with the incomplete symptom triad was proposed to be inherited in an autosomal dominant pattern of inheritance with translocation at chromosome 9p11 [21].

Malignancy was reported in six cases of MRS, half of which were hematologic. They comprised malignant lymphogranulomatosis, Hodgkin's disease, leukemic macrocheilitis, squamous cell carcinoma, retroperitoneal liposarcoma, and malignant pharyngeal lymphoma [19, 23-27].

\section{Therapy}

The good response of MRS to steroids and immunosuppressive agents is consistent with the proposed role of autoimmunity and allergy in the syndrome. Liu and Yu recommended systemic corticosteroids as the first line of treatment [13]. Rivera-Serrano et al agreed [28] with others recommending the use of intralesional injections to avoid the risk of side effects $[6,7]$. Sobjanek et al suggested that combination therapy of intralesional corticosteroids and dapsone (the former enhancing the efficacy of dapsone) was safe and effective treatment [29]. Although intralesional corticosteroid may alleviate cheilitis granulomatosa, its impact on facial palsy remains uncertain.
If all else fails, Stein et al recommended anti-tumor necrosis factor $\alpha$ agents, which they used successfully to treat a patient with non-caseating granulomatous cheilitis with neurological impairment and elevated Ig who had responded to adalimumab after failing steroids, azathioprine, and methotrexate [30]. Antibiotics with an anti-inflammatory function, (e.g., minocycline) were also used in cases of granulomatous cheilitis $[31,32]$. Ratzinger et al, who documented a good outcome with clofazimine and infliximab, recommended these agents for treating MRS with or without Crohn's disease [8]. Because of the serious side effects of biological agents, however, we recommend that they be reserved for patients in whom other agents have failed or who present with systemic involvement. Long-term follow-up visits are also recommended at this stage to observe the patient's response to treatment as well as to identify any relapses that might occur.

We suggest some guideline investigations that could be helpful for managing MRS based on the case reviews and reports we collected. They can be reviewed later, when a major trial is undertaken to study this syndrome. They are outlined in Table 2.

Finally, although MRS is a rare idiopathic disease, it is being increasingly reported. Its cause and course, however, remain unsettled. We have proposed some general guideline investigations that we hope will help identify its etiology.

\section{Ethics Approval and Consent to Participate}

It is approved by the Research and Ethics Committee in King Hamad University Hospital, Busaiteen, Kingdom of Bahrain. Documents are available.

\section{Consent for Publication}

Consent is signed by the patient for: medical care, surgical bi- 
opsy, use of material including photographs for medical education and research. Documents are available.

\section{Conflict of Interest}

We, Manal Alsabbagh and Azad Kassim, have no conflict of interest to declare.

\section{Funding}

None.

\section{Author Contributions}

Both authors contributed equally in managing the case and producing the manuscript.

\section{Abbreviations}

Ig: immunoglobulin; MRS: Melkersson-Rosenthal syndrome; OFG: orofacial granulomatosis

\section{References}

1. Woo SB. Biology and pathology of the oral cavity. In: Wolff K, Goldsmith L, Katz S, Gilchrest B, Paller AS, Leffell D, Paler A, editors. Fitzpatrick's Dermatology in General Medicine. 8th ed. New York: McGraw-Hill; 2011. p. 846-847.

2. Greene RM, Rogers RS, 3rd. Melkersson-Rosenthal syndrome: a review of 36 patients. J Am Acad Dermatol. 1989;21(6):1263-1270.

3. Tilakaratne WM, Freysdottir J, Fortune F. Orofacial granulomatosis: review on aetiology and pathogenesis. J Oral Pathol Med. 2008;37(4):191-195.

4. Jasinska D, Boczon J. Melkersson-Rosenthal syndrome as an early manifestation of mixed connective tissue disease. Eur J Med Res. 2015;20:100.

5. Chu Z, Liu Y, Zhang H, Zeng W, Geng S. Melkersson-Rosenthal Syndrome with Genitalia Involved in a 12-Year-Old Boy. Ann Dermatol. 2016;28(2):232-236.

6. Ozgursoy OB, Karatayli Ozgursoy S, Tulunay O, Kemal O, Akyol A, Dursun G. Melkersson-Rosenthal syndrome revisited as a misdiagnosed disease. Am J Otolaryngol. 2009;30(1):33-37.

7. Rachisan AL, Hrusca A, Gheban D, Cainap S, Pop TL, Baican A, Fodor L, et al. Granulomatous cheilitis of Miescher: the diagnostic proof for a Melkersson-Rosenthal syndrome. Rom J Morphol Embryol. 2012;53(3 Suppl):851-853.

8. Ratzinger G, Sepp N, Vogetseder W, Tilg H. Cheilitis granulomatosa and Melkersson-Rosenthal syndrome: evaluation of gastrointestinal involvement and therapeu- tic regimens in a series of 14 patients. J Eur Acad Dermatol Venereol. 2007;21(8):1065-1070.

9. Elias MK, Mateen FJ, Weiler CR. The MelkerssonRosenthal syndrome: a retrospective study of biopsied cases. J Neurol. 2013;260(1):138-143.

10. Morales C, Penarrocha M, Bagan JV, Burches E, Pelaez A. Immunological study of Melkersson-Rosenthal syndrome. Lack of response to food additive challenge. Clin Exp Allergy. 1995;25(3):260-264.

11. Fitzpatrick L, Healy CM, McCartan BE, Flint SR, McCreary CE, Rogers S. Patch testing for food-associated allergies in orofacial granulomatosis. J Oral Pathol Med. 2011;40(1):10-13.

12. Feng S, Yin J, Li J, Song Z, Zhao G. Melkersson-Rosenthal syndrome: a retrospective study of 44 patients. Acta Otolaryngol. 2014;134(9):977-981.

13. Liu R, Yu S. Melkersson-Rosenthal syndrome: a review of seven patients. J Clin Neurosci. 2013;20(7):993-995.

14. Degirmenci Y, Kobak S, Hayretdag Ors C, Ozisik Karaman H. Melkersson-Rosenthal syndrome in a patient with systemic lupus erythematosus: a rare comorbidity. Arch Rheumatol. 2012;27:258-261.

15. Lee YJ, Cheon CK, Yeon GM, Kim YM, Nam SO. Melkersson-Rosenthal syndrome with Hashimoto thyroiditis in a 9-year-old girl: an autoimmune disorder. Pediatr Neurol. 2014;50(5):503-506.

16. Lazzerini M, Bramuzzo M, Ventura A. Association between orofacial granulomatosis and Crohn's disease in children: systematic review. World J Gastroenterol. 2014;20(23):7497-7504.

17. Leao JC, Hodgson T, Scully C, Porter S. Review article: orofacial granulomatosis. Aliment Pharmacol Ther. 2004;20(10):1019-1027.

18. Scully C, Langdon J, Evans J. Marathon of eponyms: 13 Melkersson-Rosenthal syndrome. Oral Dis. 2010;16(7):707-708.

19. Freeman GL. Complement abnormality in MelkerssonRosenthal. Allergy. 2004;59(3):362.

20. Masson F, Barete S, Fremeaux-Bacchi V, Szpirglas H, Agbo-Godeau S, Chosidow O, Piette JC, et al. Melkersson-Rosenthal syndrome and acquired $\mathrm{C} 1$ inhibitor deficiency. Dermatology. 2008;217(2):114-120.

21. Troiano G, Dioguardi M, Giannatempo G, Laino L, Testa NF, Cocchi R, De Lillo A, et al. Orofacial granulomatosis: clinical signs of different pathologies. Med Princ Pract. 2015;24(2):117-122.

22. Xu XG, Guan LP, Lv Y, Wan YS, Wu Y, Qi RQ, Liu ZG, et al. Exome sequencing identifies FATP1 mutation in Melkersson-Rosenthal syndrome. J Eur Acad Dermatol Venereol. 2017;31(5):e230-e232.

23. Bardadin T, Sliwowska W. Case of Melkersson-Rosenthal syndrome in a patient with malignant lymphogranulomatosis. Przegl Dermatol. 1966;53(2):167-170.

24. Mulvihill JJ, Eckman WW, Fraumeni JF, Jr., Dryden RM, Young RC. Melkersson-Rosenthal syndrome, Hodgkin disease, and corneal keratopathy. Arch Intern Med. 1973;132(1):116-117.

25. Connelly TJ, Kauh YC, Luscombe HA, Becker G. Leukemic macrocheilitis associated with hairy-cell leukemia 
and the Melkersson-Rosenthal syndrome. J Am Acad Dermatol. 1986;14(2 Pt 2):353-358.

26. Pretel Serrano L, Torralba Saura M, Herrero Huerta F, Alonso Lajara JD. [A late Melkersson-Rosenthal syndrome and retroperitoneal liposarcoma]. Rev Clin Esp. 1992;190(5):283.

27. Kanda A, Kanekura T, Ohtake N, Kanzaki T. MelkerssonRosenthal syndrome with malignant pharyngeal lymphoma. J Dermatol. 1996;23(9):658-659.

28. Rivera-Serrano CM, Man LX, Klein S, Schaitkin BM. Melkersson-Rosenthal syndrome: a facial nerve center perspective. J Plast Reconstr Aesthet Surg. 2014;67(8):1050-1054.

29. Sobjanek M, Michajlowski I, Zelazny I, Medrzycka-Dabrowska W, Wlodarkiewicz A. What is the most effec- tive treatment of cheilitis granulomatosa in MelkerssonRosenthal syndrome? J Eur Acad Dermatol Venereol. 2010;24(3):364-365.

30. Stein J, Paulke A, Schacher B, Noehte M. An extraordinary form of the Melkersson-Rosenthal syndrome successfully treated with the tumour necrosis factor-alpha blocker adalimumab. BMJ Case Rep. 2014;2014.

31. Banks T, Gada S. A comprehensive review of current treatments for granulomatous cheilitis. Br J Dermatol. 2012;166(5):934-937.

32. Stein SL, Mancini AJ. Melkersson-Rosenthal syndrome in childhood: successful management with combination steroid and minocycline therapy. J Am Acad Dermatol. 1999;41(5 Pt 1):746-748. 